\title{
Tomoceridae (Collembola, Entomobryomorpha) from the southern Annamitic cordillera: redescription of Tomocerus ocreatus Denis, 1948 and description of a new species of Tomocerina Yosii, 1955
}

\author{
Daoyuan YU ${ }^{1, *}$, Le Cong MAN ${ }^{2} \&$ Louis DEHARVENG $^{3}$ \\ ${ }^{1}$ Soil Ecology Lab, College of Resources and Environmental Sciences, \\ Nanjing Agricultural University, Nanjing 210095, China. \\ ${ }^{2}$ Department of Biology, University of Natural Sciences, \\ Ho Chi Minh City National University, Vietnam. \\ ${ }^{3}$ Institut de Systématique, Evolution, Biodiversité, ISYEB - UMR 7205 - CNRS, MNHN, UPMC, \\ EPHE, Muséum national d'Histoire naturelle, Sorbonne Universités, \\ 57 rue Cuvier, CP 50, 75005 Paris, France. \\ *Corresponding author. dyyu1987@,hotmail.com; yudy@njau.edu.cn \\ ${ }^{2}$ Email: tranletamlinh@yahoo.com.vn \\ ${ }^{3}$ Email: deharven@mnhn.fr

\footnotetext{
${ }^{1}$ urn:Isid:zoobank.org:author:07321441-2070-4DB2-BC59-D3F1C8300BC3

${ }^{2}$ urn:lsid:zoobank.org:author:0D7A5067-F6EF-456F-8B32-D1B61DCC4CDD

${ }^{3}$ urn:1sid:zoobank.org:author:D8F5C679-C30C-442C-8621-D3B8EDB17EF7
}

\begin{abstract}
Two species of Tomoceridae were found near Dalat, southern Vietnam. Tomocerus ocreatus Denis, 1948 is redescribed based on a neotype specimen. Previous records of Tomocerus ocreatus in non-type localities are reevaluated. A new species Tomocerina annamitica sp. nov. is described. The new species is mainly characterized by its small body size, pointed tenent hair, compound dental spines and the absence of intermediate teeth on mucro.
\end{abstract}

Key words. Vietnam, Southeast Asia, Tomocerinae, taxonomy, neotype.

Yu D., Man L.C. \& Deharveng L. 2016. Tomoceridae (Collembola, Entomobryomorpha) from the southern Annamitic cordillera: redescription of Tomocerus ocreatus Denis, 1948 and description of a new species of Tomocerina Yosii, 1955. European Journal of Taxonomy 176: 1-14. http://dx.doi.org/10.5852/ejt.2016.176

\section{Introduction}

The family Tomoceridae contains at least 169 known species (Bellinger et al. 1996-2015) distributed worldwide. In Asia Tomoceridae are abundant in the north but have only a few reports in the south. Three species were previously described from Vietnam: Tomocerus ocreatus Denis, 1948 from Dalat, Lam Dong Province; Tomocerus nodentalis Nguyen, 1995 from Moc Chau, Son La Province; and Tomocerus postantennalis Yu, Zhang \& Deharveng, 2014 from Na Hang, Tuyen Quang Province. Tomocerus ocreatus is characterized by the shape and arrangement of dental spines. Tomocerus nodentalis is very 
peculiar for the absence of dental spines, which character is only observed in very early instars of other species (Goto 1956; Uchida \& Chiba 1958). Tomocerus postantennalis is a cave species with developed postantennal organs in adult stage. To the present knowledge, Tomocerus nodentalis and Tomocerus postantennalis are both endemic species, whereas Tomocerus ocreatus is known to be the most widely distributed Asian species of Tomocerus Nicolet, 1842. Besides its type locality, Tomocerus ocreatus has records from Himalaya (Yosii 1966), Japan (Yosii 1956, 1967, 1969, 1977; Chiba 1968), Korea (Yosii \& Lee 1963; Lee 1974, 1975), China (Stach 1964; Liu et al. 1998), northern Vietnam (Stach 1965), eastern Russia (Sakhalin island) (Martynova 1977), etc. But intraspecific differences were often reported by descriptions of non-type Tomocerus ocreatus. For instance, Chiba (1968) distinguished three varieties from Japanese Tomocerus ocreatus according to the variation in the body size and relative length of body parts. A molecular study (Zhang et al. 2014) also revealed "cryptic" diversity among Chinese specimens previously identified as Tomocerus ocreatus.

To clarify the true status and distribution of Tomocerus ocreatus, a redescription of its type specimen is required, but the holotype and only specimen of the species has been lost in the Muséum national d'Histoire naturelle (Paris) where the Denis' collection is currently stored. In a recent sample collected from Dalat (Lam Dong Province) in 2008, we have found a specimen that matches well the original description in morphology. We designate this specimen as neotype of Tomocerus ocreatus.

A new tomocerid species was found in the same sample as Tomocerus ocreatus. We assign the new species to Tomocerina Yosii, 1955 because it has no toothlet on the outer basal mucronal tooth. To our knowledge, Tomocerina annamitica sp. nov. is so far the southernmost record of the genus.

\section{Materials and methods}

Specimens were collected with aspirators or Berlese funnels. Photographs were taken under a Jenoptik ProgRes C10+ camera mounted on a Leica MZ 16 stereomicroscope. Specimens were cleared in lactic acid and mounted in Hoyer's solution (Krantz 1978). For some specimens the head, the furca and the legs were cut off from the trunk and mounted separately for detailed observation. The slide-mounted specimens were studied using a Leica DMLB microscope or a Nikon $80 \mathrm{i}$ microscope.

We follow Fjellberg (2007) for maxillary lamellae numbering, Yu et al. (2014) for the pattern of cephalic dorsal chaetotaxy and Christiansen (1964) for body macrochaetotaxy. The description of the body chaetotaxy refers to one side only since in most case it is symmetric. The exact morphology of each chaeta was uncertain due to shedding. The dental spines formula follows that of Folsom (1913), in which the dental spines are arranged from basal to distal, with a slash indicating the separation between basal and medial subsegments and the Roman numerals referring to spines that are noticeably larger. If not mentioned specially, all descriptions are based on fully developed individuals.

\section{Abbreviations}

Ant. = antennal segment

$\mathrm{PAO}=$ postantennal organ

Th. $=$ thoracic segment

Abd. $=$ abdominal segment

Institutional acronyms:

HCMCU $=$ Ho Chi Minh City National University, Vietnam

MNHN = Muséum national d'Histoire naturelle, Paris, France

NJAU = Nanjing Agricultural University, Nanjing, China 


\section{Results}

Class Collembola Lubbock, 1873

Order Entomobryomorpha Börner, 1913

Superfamily Tomoceroidea Szeptycki, 1979

Family Tomoceridae Börner, 1913

Subfamily Tomocerinae Schäffer, 1896

Genus Tomocerus Nicolet, 1842

\section{Diagnosis}

Moderate to large sized Tomocerinae, usually longer than $3 \mathrm{~mm}$; body colour pale to dark, some species with distinct colour pattern; eyes at most $6+6$; trochantero-femoral organ reduced to 1,1 chaetae; dens of furca basally without outer strong chaetae or inner large differentiated scales; shape of dental spines from simple to compound among different species; mucro with two dorsal lamellae and two basal teeth, outer basal tooth with corner toothlet. Commonest group of Tomocerinae in Eurasia Continent, especially abundant in East Asia.

Tomocerus ocreatus Denis, 1948

Figs 1A, 2, 3

\section{Diagnosis}

Typical Tomocerus species with pale body colour, moderately long antennae and full set of $6+6$ eyes. Head without distinct PAO; Th. II with relatively reduced number of macrochaetae; tenent hair clavate, moderately developed; unguis with 5-6 teeth; tenaculum unscaled, with numerous chaetae; manubrium without dorsal scales and blunt prominent chaetae; dental spines compound with numerous moderate sized denticles; no small dental spine between two distal large spines; mucro with 9-10 intermediate teeth.

\section{Neotype}

VIETNAM: + , on slide. Collected in Hon Giao, Bi Doup massif, northeast of Dalat, Lam Dong Province, $108^{\circ} 42^{\prime} 53^{\prime}$ 'E, $12^{\circ} 11^{\prime} 11^{\prime \prime} \mathrm{N}$, alt. $1630 \mathrm{~m}, 12$ Jun. 2008, by Louis Deharveng \& Anne Bedos (sample code Vn08-150). Deposited in MNHN (specimen Vn08-150_To1).

\section{Description}

Body length $3.6 \mathrm{~mm}$. Ground colour uniformly yellowish white. Ant. III+IV, antero-ventral part of head, coxae and tibiotarsi with dark pigment. Eye patches black. Scales brown (Fig. 1A).

Body densely clothed by scales and various types of chaetae. Scales of typical morphology of Tomocerinae, with continuous longitudinal ridges on surface (Lubbock 1873). Ordinary chaetae of different sizes. Microchaetae smooth and pointed. Macrochaetae and mesochaetae from slightly to strongly ciliated, some slightly ciliated mesochaetae appearing to be smooth under optical microscope. Most macrochaetae straight, rod-like and subcylindrical, some macrochaetae on posterior abdominal segments long, curved and acuminate. Mesochaetae acuminate, shorter and thinner than macrochaetae. S-chaetae subcylindrical, more hyaline than ordinary chaetae, as small as microchaetae except long ones on Abd. IV. Dorso-inner chaetae on dens modified as strong pointed spines. Pseudopores as small circular structures similar to chaetae sockets, distributed at least on Th. II to Abd. IV, coxae, and manubrium.

PAO not seen. Eyes $6+6$. Antennae nearly as long as body. Antenna length ratio as I:II:III $+\mathrm{IV}=$ 1.0:1.7:15.4. Ant. I and Ant. II dorsally scaled, Ant. III+IV unscaled. Prelabral and labral chaetae (labral 
formula) $4 / 5,5,4$, the distal 4 chaetae stronger. Distal edge of labrum with four curved spine-like papillae (Fig. 2A), and a well developed ventro-distal brush. Mandibular head asymmetrical, the left one with 4 teeth (including a basal rounded one) and the right one with 5 , left molar plate distally with a tapered tooth (Fig. 2B). Basal teeth of maxillary lamella 5 slightly longer than apical ones, without beard-like appendage (Fig. 2C). Maxillary outer lobe with trifurcate palp, one basal chaeta and 4 sublobal hairs (Fig. 2D). Both dorsal and ventral sides of head scaled. Cephalic dorsal macrochaetotaxy: anterior area: 2, 2; interocular area: 2, 6, central uneven macrochaeta absent; postocular area: $2+2$; posterior area: 2 . Posterior margin of head with about $20+20$ small chaetae (Fig. 2E). Mentum with 5 chaetae, submentum with numerous chaetae.

Pattern of body chaetotaxy as in Fig. 2F. Bothriotricha 2, 1/0, 0, 1, 2, 0, 0 on Th. II-Abd. VI, respectively, as typical in Tomocerinae. Macrochaetae densely arranged along anterior margin of Th. II (not shown in figure). Th. II with a row of macrochaetae behind anterior margin. Number of macrochaetae or large mesochaetae in the posterior row as 3, 3/3, 3, 4, 2, 4 (3 dorsal+1 lateral) from Th. II to Abd. V. On Th. II no macrochaeta near the pseudopore contrary to most other tomocerids; on Abd. III s-microchaeta and accompanying microchaeta posterior to lateral macrochaeta; Abd. IV with one lateral macrochaeta and numerous long s-chaetae; on Abd. V inner macrochaeta smaller than others in posterior row; Abd. VI with numerous chaetae of moderate size. Most mesochaetae laterally and posteriorly on terga. Pseudopores near the axis of terga, 1, 1/1, 1, 1, 1, 0, 0 from Th. II to Abd. VI.

Legs with numerous ordinary chaetae. Trochantero-femoral organ with 1, 1 slender chaetae. Front, middle and hind tibiotarsus dorsally with 1, 1, 3 long prominent chaetae, ventrally with 4-5, 5, 6 blunt spine-like chaetae (Fig. 2G). Each tibiotarsus with a distal whorl of 11 chaetae, ventral 6 as ordinary chaetae, dorsal 5 modified: tenent hair clavate, as long as inner edge of unguis; 2 accessory chaetae extremely minute; 2 guard chaetae thin and long, slightly shorter than tenent hair (Fig. 3A, B). Unguis

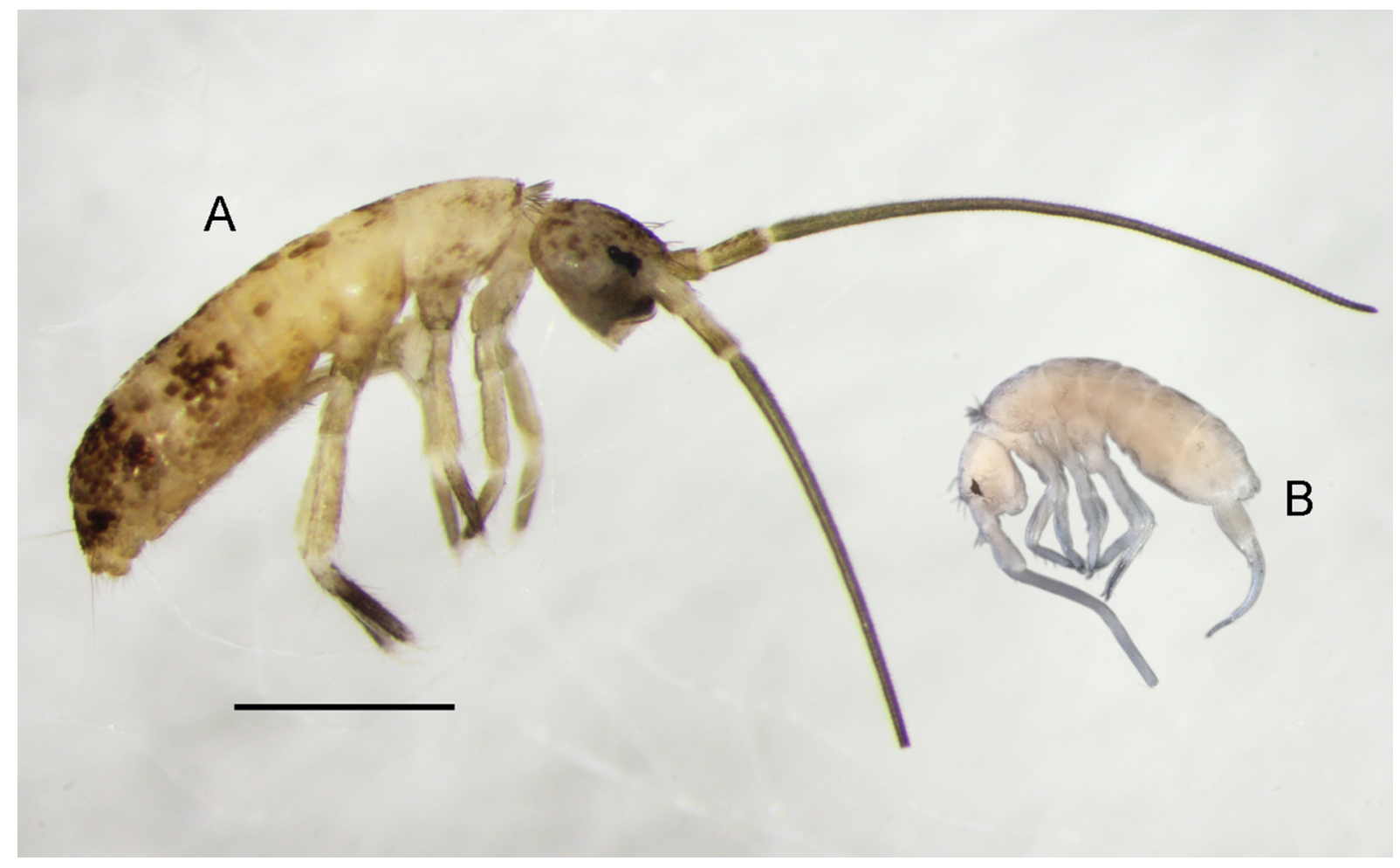

Fig. 1. Appearance of Tomocerus ocreatus Denis, 1948 and Tomocerina annamitica sp. nov. in alcohol. A. Tomocerus ocreatus (lateral view). B. Tomocerina annamitica sp. nov. (lateral view). Scale bar: $1 \mathrm{~mm}$. 
slender, with baso-internal ridging visible in lateral view; lateral teeth pointed, of moderate size. Inner edge of unguis with 5-6 teeth, the basal tooth smaller, the other subequal in size. Unguiculus about half as long as unguis, its inner edge with 1 tooth. Pretarsal chaetae 1+1, much larger than tenent-hair accessory chaetae (Fig. 3B).
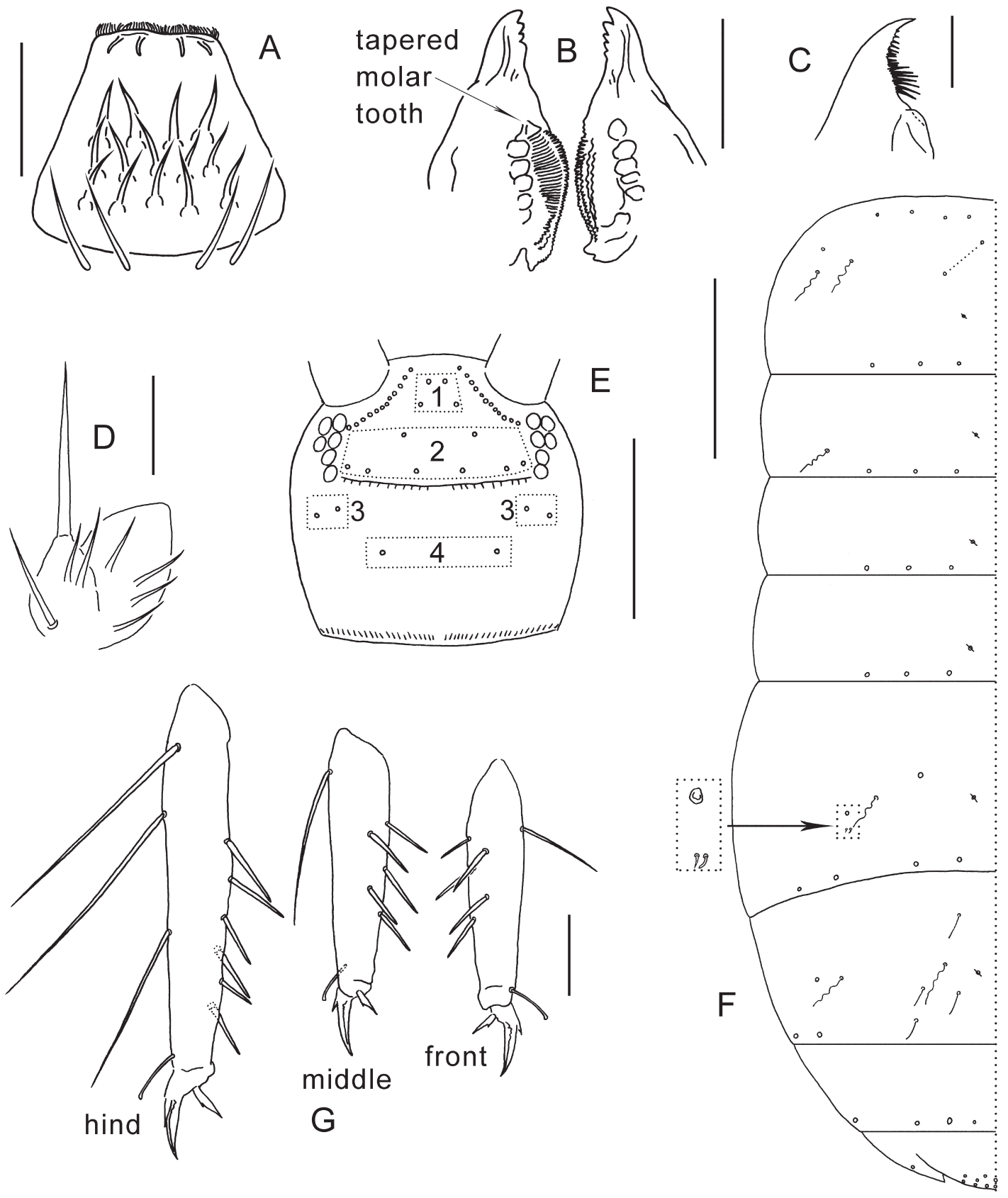

Fig. 2. Tomocerus ocreatus Denis, 1948. A. Labrum (dorsal view). B. Mandibular head (dorsal view). C. Lamella 5 of maxillary head (dorsal view). D. Maxillary outer lobe (ventral view). E. Cephalic dorsal chaetotaxy (circles $=$ sockets of chaetae, same as below; $1=$ anterior area; $2=$ interocular area; $3=$ postocular area; $4=$ posterior area). F. Dorsal chaetotaxy of Th. II-Abd. VI (circle with a slash $=$ pseudopore, same as below). G. Right tibiotarsi (lateral view). Scale bars: A, B, G $=100 \mu \mathrm{m} ; \mathrm{C}=10 \mu \mathrm{m}$; $\mathrm{D}=50 \mu \mathrm{m} ; \mathrm{E}, \mathrm{F}=500 \mu \mathrm{m}$. 
Ventral tube with scales on both anterior and posterior faces, lateral flap unscaled, anterior face with about 15 chaetae on each side, posterior face with about 55 chaetae, each lateral flap with 45-47 chaetae. Rami of tenaculum with $4+4$ teeth, anterior face with 13 small chaetae and without scale (Fig. 3C). Furca ratio manubrium: dens: mucro $=2.9-3.1: 4.1-4.4: 1.0$. Manubrium ventrally scaled, without chaetae, laterally with large round scales and 11 chaetae, proximal 2 chaetae small and slender, distal 9 distinctly stronger and serrated; dorsal scales absent; each dorsal chaetal stripe with about 90 chaetae in different sizes, without blunt chaetae; pseudopores 11-12 on each side (Fig. 3D); external corner chaeta as large as small mesochaetae in chaetal stripe (Fig. 3E). Dens basally without inner modified scale or outer
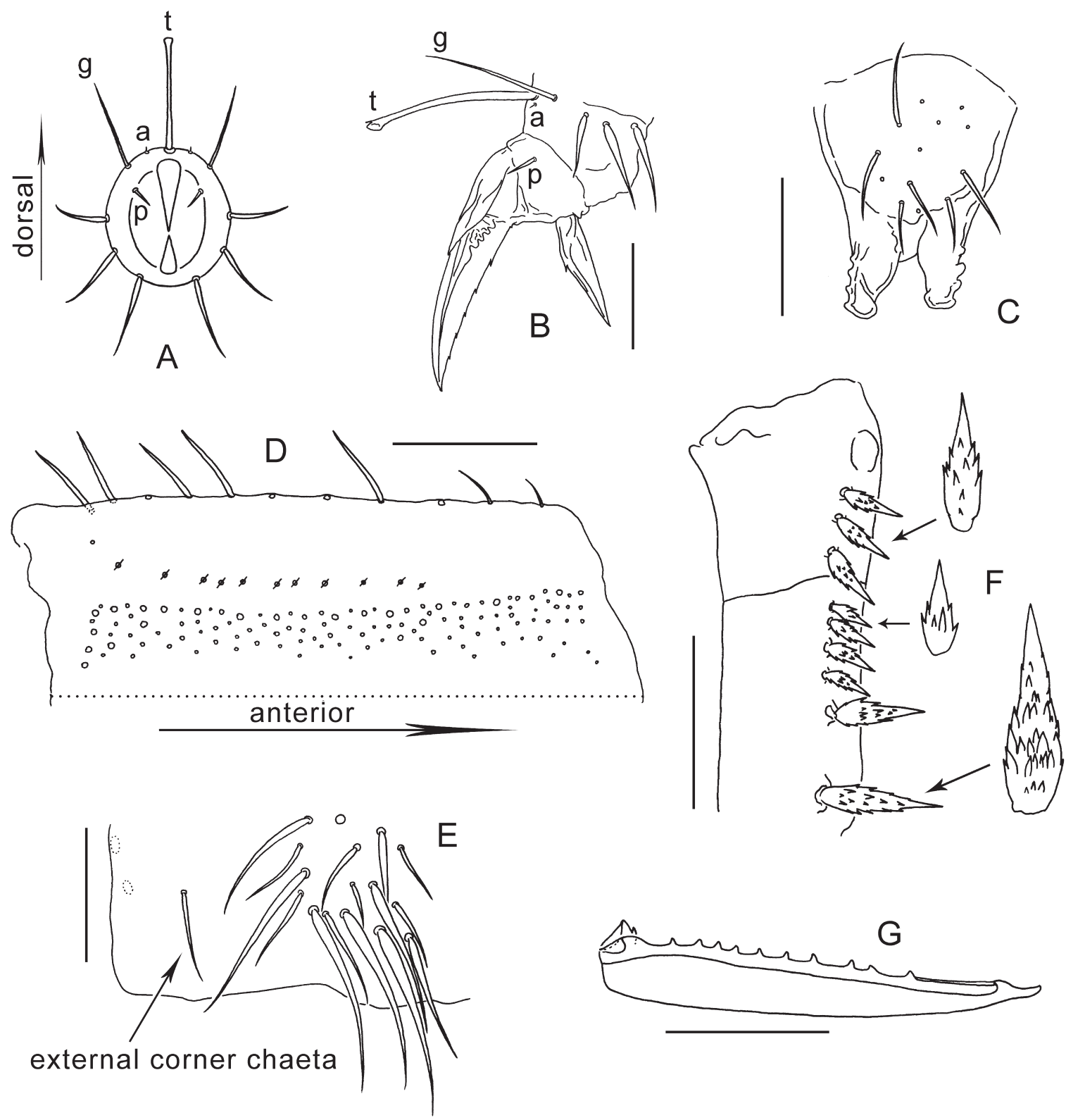

Fig. 3. Tomocerus ocreatus Denis, 1948. A. Diagram of tibiotarsal distal chaetae (distal view; $\mathrm{t}=$ tenent hair; $\mathrm{a}=$ accessory chaeta; $\mathrm{g}=$ guard chaeta; $\mathrm{p}=$ pretarsal chaeta, same as below). B. Hind claw (lateral view). C. Tenaculum (anterior view). D. Left side of manubrium (dorsal view). E. Disto-external corner of manubrium (dorsal view). F. Basal and middle subsegments of dens with dental spines (dorsal view). G. Mucro (dorso-inner view). Scale bars: $\mathrm{B}, \mathrm{C}, \mathrm{E}, \mathrm{G}=50 \mu \mathrm{m} ; \mathrm{D}, \mathrm{F}=100 \mu \mathrm{m}$. 
strong chaetae. Dental spines formula as 3/4, II, distal spine strongest, about 0.1 times as long as dens; all spines with numerous denticles of moderate size (Fig. 3F). Dens dorsally with ordinary chaetae and feather-like chaetae as typical in Tomocerinae, ventrally with only scales. Mucro elongated, bearing numerous smooth chaetae with elongated sockets; both basal teeth with proximal lamellae, the outer tooth with a toothlet; apical and subapical tooth subequal; structure of dorsal lamellae of Tomocerus type, two dorsal lamellae running from subapical tooth, outer lamella ending in inner basal tooth, inner lamella ending freely at base of mucro; outer lamella with 9-10 subequal intermediate teeth (Fig. 3G).

\section{Remarks}

Denis (1948) provided accurate description for Tomocerus ocreatus. For instance, he described clearly the dental spines as "covered with secondary spines, more similar to scales than denticles", and the figure showed that those scale-like denticles were of moderate size and covered at least basal half of each spine, on which account we rejected some records of non-type Tomocerus ocreatus (see below). But naturally, some later revealed characters were not mentioned by him. For instance, chaetotaxy had not been used for taxonomy in Tomocerinae before Yosii (1956). The limit of original description is a main reason for the misidentification of Tomocerus ocreatus in some subsequent records, and finally turned the species into a complex including a number of closely related forms from Vietnam to eastern Russia. To overcome the deep confusion about this species, a redescription of type specimen is necessary. Because the holotype and unique type specimen of Tomocerus ocreatus was lost, we propose to set up a neotype for the species from specimens of the type locality.

Denis (1948) described Tomocerus ocreatus on one specimen, from "plateau du Lang Biang, $2400 \mathrm{~m}$. alt., forêt tropicale". It is not the Lang Bian peak itself that reaches $2400 \mathrm{~m}$ but the Chu Yang Sin massif north of the Lang Bian (= Dalat) plateau, the Lang Bian peak being only $2197 \mathrm{~m}$ in altitude. The Chu Yang Sin massif was and still is of very difficult access, and was certainly not the place where the collector, Dawydoff, operated. There are two main patches of subtropical forests around Dalat: a small one on the Lang Bian peak, and a much larger one $25 \mathrm{~km}$ northeast on the Bi Doup massif, which reaches $2287 \mathrm{~m}$ and is less easy to access. In any case, it is most likely on the slopes of one of these massifs that Tomocerus ocreatus was collected. On this account, we propose to designate a specimen that we obtained from litter sample in the Bi Doup mountain as the neotype.

The neotype is highly identical with the original description in almost all described characters, including the body colour, the length of antennae, the structure of claws and the morphology and arrangement of dental spines. The only difference is that in the neotype specimen the numbers of ungual teeth, dental spines and mucronal teeth are slightly larger than in the original one. It could happen in Tomoceridae that within the same species larger individuals have a few more teeth and dental spines than smaller ones, so the slight difference mentioned above can be treated as intraspecific considering the neotype specimen is slightly larger than the lost holotype ( $3.6 \mathrm{~mm}$ versus $3.3 \mathrm{~mm}$ ).

Regarding previous non-type records of Tomocerus ocreatus, Stach $(1964,1965)$ redescribed two different forms of Tomocerus ocreatus from southeastern China and northern Vietnam, respectively, and also claimed that the Japanese record of "Tomocerus minor" by Uchida (1953) was actually Tomocerus ocreatus; Yosii described a Japanese species Tomocerus kawamurai (Yosii, 1954) and then synonymized it with Tomocerus ocreatus (Yosii 1956, 1967); Chiba (1968) recognized three forms in Japanese Tomocerus ocreatus; Lee (1975) made descriptive notes on Korean specimens; Martynova (1977) recorded Tomocerus ocreatus in Sakhalin and made some descriptive notes on it. These redescriptions had subtle to considerable differences to each other, and were never identical to the original description. The Chinese "ocreatus" recorded in Hangzhou ("Hangchow"), Zhejiang Province by Stach (1964) has brownish body colour and distinctly short antenna about half the length of body; the northern Vietnamese record from Lao Cai Province by the same author (Stach 1965) bears a small dental spine between two large distal spines, and the denticles 
on spines are finer than those in the original description, therefore it is more similar to Tomocerus folsomi Denis, 1929 from Yunnan Province, China; the Japanese "ocreatus" described by Yosii (1967) has dorsal scales and $2+2$ blunt principal chaetae on manubrium which are absent in the true Tomocerus ocreatus; other records (Chiba 1968; Lee 1975; Martynova 1977) all have dental spines with one or two whorls of crownlike denticles near the base which is not the morphology of the true ocreatus form. But these remarkable differences were treated as intraspecific variations when other characters showed high similarity. Inferred from the present morphological review and the molecular study on Chinese ocreatus complex (Zhang et al. 2014), the aforementioned non-type "ocreatus" may each represent an independent species in the complex, and so far the true Tomocerus ocreatus is known as only endemic to southern Annamitic range in Vietnam.

\section{Genus Tomocerina Yosii, 1955}

\section{Diagnosis}

Small to moderate sized Tomocerinae, usually less than $2 \mathrm{~mm}$ in length; body colour pale to light grey, seldom dark; antennae usually much shorter than body; eyes at most $6+6$; trochantero-femoral organ with 1,1 or more chaetae; dens of furca basally without outer strong chaetae or inner large differentiated scales; shape of dental spines from simple to compound; mucro with two dorsal lamellae and two basal teeth, outer basal tooth without corner toothlet. Widely distributed in Northern Hemisphere.

\section{Tomocerina annamitica sp. nov. urn:1sid:zoobank.org:act:9AD947EC-5A05-4385-9A92-8B0DC590A94D}

Figs 1B, 4, 5

\section{Diagnosis}

Tomocerina species with typical body size and pigmentation; antennae about 0.8 times as long as body, relatively long for Tomocerina; Th. II with only one bothriotrichum; tibiotarsi with multiple strong chaetae; tenet hair small and pointed; unguis with 1-2 inner teeth; dental spines compound with denticles; only one distal spine distinctly larger; mucro without intermediate tooth.

\section{Etymology}

Specific name derived from its type locality: the southern Annamitic range.

\section{Type material}

\section{Holotype}

VIETNAM: 9 , on slide. Collected in Hon Giao, Bi Doup massif, northeast of Dalat, Lam Dong Province, $108^{\circ} 42^{\prime} 53^{\prime}$ 'E, $12^{\circ} 11^{\prime} 11^{\prime}$ 'N, alt. 1630 m, mixed forest litter, 12 Jun. 2008, by Louis Deharveng \& Anne Bedos (sample code Vn08-150), deposited in MNHN (specimen Vn08-150_Ta1).

Paratypes

VIETNAM: 2 q 9 , on slides, same data as holotype, 1 in HCMCU (specimen Vn08-150_Ta2) and 1 in NJAU (specimen Vn08-150_Ta3).

\section{Other material examined}

VIETNAM: 5 specimens on slides and 28 specimens in alcohol, collected in the Hon Ba Nature Reserve, Kanh Hoa Province, located $25 \mathrm{~km}$ east-southeast of Hon Giao and $25 \mathrm{~km}$ south-southwest of Nha Trang, 108 $56^{\prime} 55^{\prime \prime}-108^{\circ} 58^{\prime} 51^{\prime \prime} \mathrm{E}, 12^{\circ} 06^{\prime} 35^{\prime \prime}-12^{\circ} 07^{\prime} 09^{\prime}$ 'N, mixed forest litter, Nov. 2013, by Louis Deharveng \& Anne Bedos: alt. 850 m, Vn13-072 (3 in alcohol); 890 m, Vn13-126 (2 in alcohol), Vn13135 (4 in alcohol); $1050 \mathrm{~m}, \mathrm{Vn13-054}$ (2 9 웅, on slides), Vn13-057 (1 0 and 1 + , on sildes), Vn13-114 (1 on slide, sex undetermined), Vn13-120 (8 in alcohol); $1350 \mathrm{~m}, \mathrm{Vn13-039}$ (5 in alcohol); $1500 \mathrm{~m}$, Vn13-231 (6 in alcohol). 


\section{Description}

Body length 1.6-1.8 mm. Body colour light grey, antennae light purple, eye patches black, scales brown (Fig. 1B). Clothing of Tomocerinae type, similar to that of Tomocerus ocreatus.

PAO not seen. Eyes $6+6$. Antenna about $0.75-0.90$ times as long as body. Antenna length ratio I:II:III: $\mathrm{IV}=1.0: 1.3-1.5: 5.5-6.0: 2.2-2.5$, Ant. I and Ant. II dorsally scaled, Ant. III and Ant. IV unscaled. Labral formula 4/5,5, 4, the distal 4 chaetae stronger. Distal edge of labrum with four curved spine-like papillae, ventro-distal brush well developed. Mandibular head asymmetrical, the left one with 4 teeth (including a

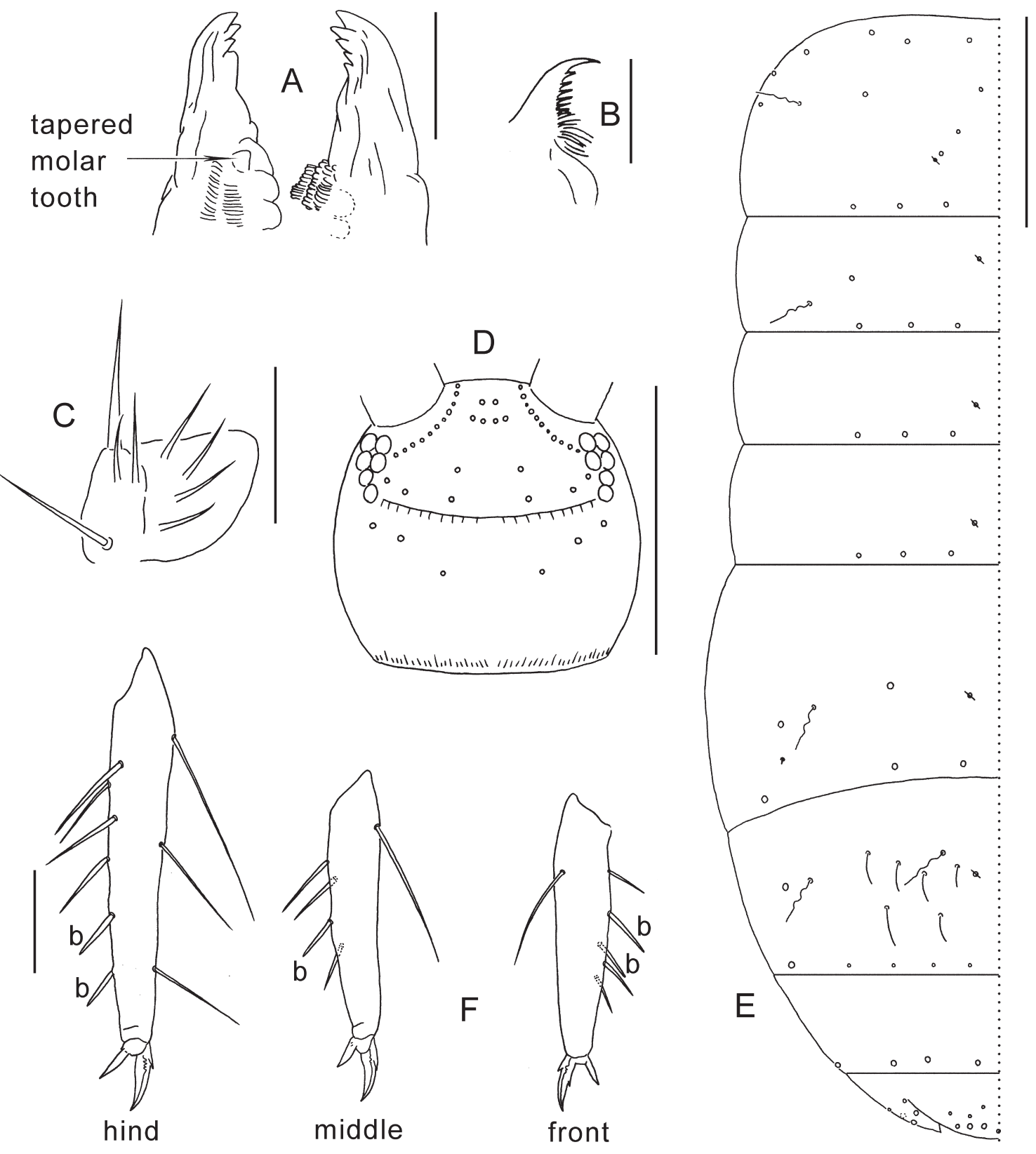

Fig. 4. Tomocerina annamitica sp. nov. A. Apical half of mandibular head (dorsal view). B. Lamella 5 of maxillary head (dorsal view). C. Maxillary outer lobe (ventral view). D. Cephalic dorsal chaetotaxy. E. Dorsal chaetotaxy of Th. II-Abd. VI. F. Left tibiotarsi (lateral view, $b=$ blunt inner chaeta). Scale bars: $\mathrm{A}, \mathrm{C}=50 \mu \mathrm{m} ; \mathrm{B}=10 \mu \mathrm{m} ; \mathrm{D}, \mathrm{E}=250 \mu \mathrm{m} ; \mathrm{F}=100 \mu \mathrm{m}$. 
basal rounded one) and the right one with 5, left molar plate distally with tapered tooth (Fig. 4A). Basal teeth of maxillary lamella 5 elongated without beard-like appendage (Fig. 4B). Maxillary outer lobe with trifurcate pulp, basal chaeta and 4 sublobal hairs (Fig. 4C). Cephalic dorsal chaetotaxy: anterior area: 2, 4; interocular area: 2, 6, central chaeta absent; postocular area: $2+2$; posterior area: 2; posterior margin: about 20+20 small chaetae of different sizes (Fig. 4D). Both dorsal and ventral side of head scaled. Mentum with 5 chaetae, submentum with numerous chaetae.

Pattern of body chaetotaxy as Fig. 4E. 1, 1/0, 0, 1, 2, 0, 0 bothriotricha on Th. II-Abd. VI. Macrochaetae densely along anterior margin of Th. II (not shown in figure). Th. II with a row of macrochaetae behind anterior margin. Number of macro- or large mesochaetae in the posterior row as 3, 3, 3, 3, 3, 5, 4 ( 3 dorsal+1 lateral) from Th. II to Abd. V. On Th. II pseudopore near posterior macrochaeta of the 3 central ones; Th. III with anterior macrochaeta; on Abd. III s-microchaeta posterior to lateral macrochaetae; Abd. IV with antero-lateral macrochaeta and numerous long s-chaetae, 4 inner mesochaetae in posterior
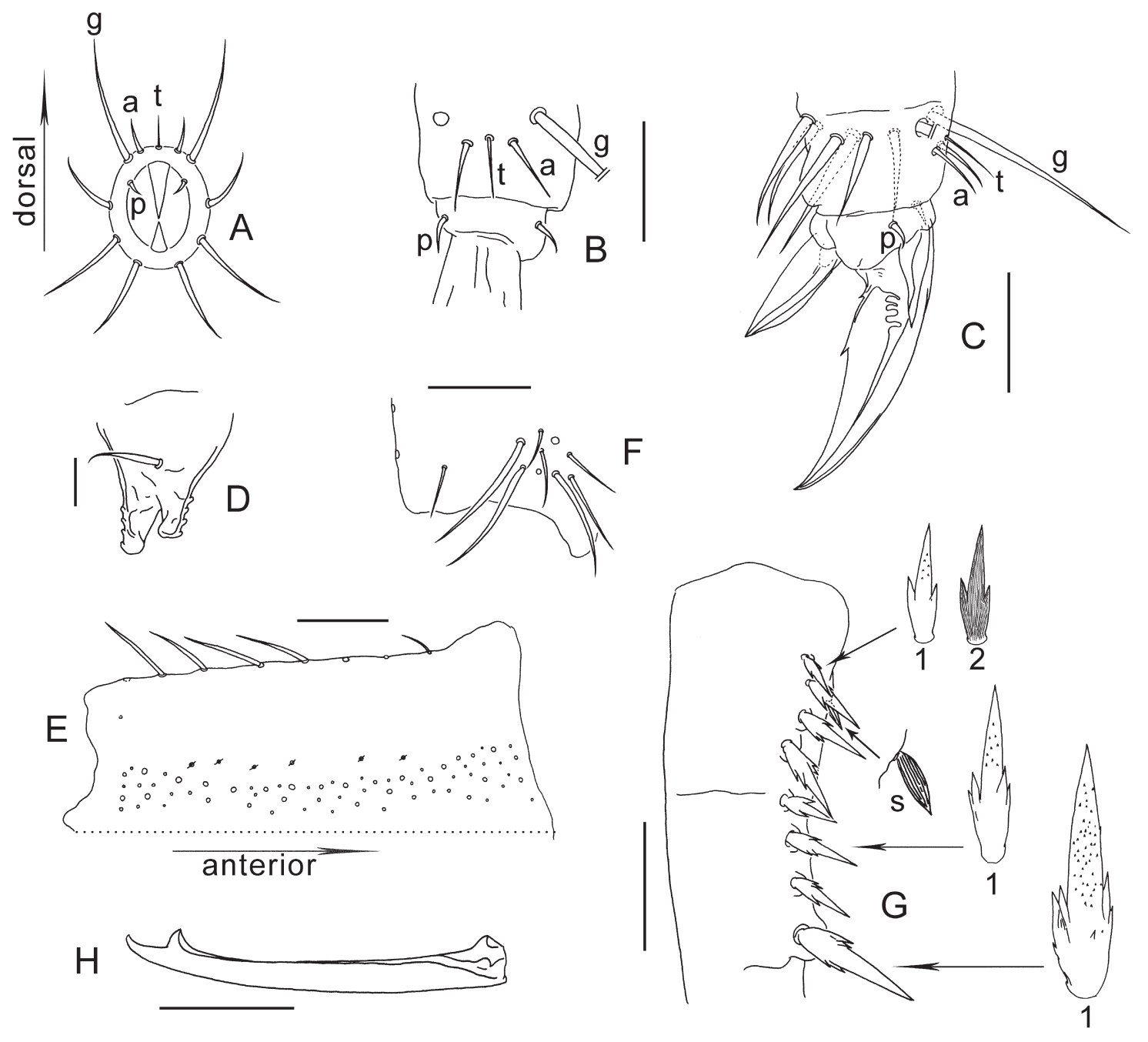

Fig. 5. Tomocerina annamitica sp. nov. A. Diagram of tibiotarsal distal chaetae (distal view). B. Tibiotarsal distal chaetae (dorsal view). C. Hind claw (lateral view). D. Tenaculum (anterior view). E. Left side of manubrium (dorsal view). F. Disto-external corner of manubrium (dorsal view). G. Basal and middle subsegments of dens with dental spines (dorsal view, $1=$ ventral side of spine, showing only distal serrations; 2 = dorsal side of spine, showing fine longitudinal ribs; $s=$ small inner scale). $\mathbf{H}$. Mucro (inner view). Scale bars: $\mathrm{B}-\mathrm{D}=20 \mu \mathrm{m} ; \mathrm{E}-\mathrm{H}=50 \mu \mathrm{m}$. 
Table 1. Discrimination between Tomocerina annamitica sp. nov. and Tomocerina purpurithora Liu, Hou \& Li, 1999.

\begin{tabular}{lcccc}
\hline \multicolumn{1}{c}{ Species } & $\begin{array}{c}\text { Number of tibiotarsal } \\
\text { strong inner chaetae }\end{array}$ & $\begin{array}{c}\text { Number of chaetae } \\
\text { on tenaculum }\end{array}$ & $\begin{array}{c}\text { Dental spines } \\
\text { formula }\end{array}$ & $\begin{array}{c}\text { Number of intermediate } \\
\text { teeth on mucro }\end{array}$ \\
\hline T. annamitica sp. nov. & $5-7,4,5-6$ & 1 & $4 / 3, \mathrm{I}$ & 0 \\
T. purpurithora & $0,0,1$ & 5 & $5 / 7,1$ & $5-7$ \\
\hline
\end{tabular}

row prominent but smaller than outer macrochaeta; on Abd. VI dorsal flap with $2+2$ and lateral flap with 3 macrochaetae. Most mesochaetae laterally and posteriorly on terga. Pseudopores near the axis of terga, $1,1 / 1,1,1,1,0,0$ from Th. II-Abd. VI.

Legs with numerous ordinary chaetae. Trochantero-femoral organ with 1,1 chaetae. Tibiotarsi dorsally with $1,1,3$ prominent chaetae from front to hind leg, ventrally with 5-7, 4, 5-6 strong chaetae, only 2, 1, 2 of them blunt (Fig. 4F). Each tibiotarsus with a distal whorl of 11 chaetae, the dorsal 5 modified: tenent hair pointed, short and thin; 2 accessory chaetae about as long as but thicker than tenent hair, and longer than pretarsal chaetae; 2 outer guard chaetae strong, as long as inner edge of unguis (Fig. 5A-C). Scales absent from front and mid tibiotarsi but present on hind tibiotarsus. Unguis slender, lateral teeth of moderate size, internal ridging present. Inner edge of unguis with a small basal and 0-1 larger more distal teeth. Unguiculus about half as long as unguis, without tooth on inner or outer edge. Pretarsus with $1+1$ chaetae (Fig. 5C).

Ventral tube anteriorly with a few small scales, each side with 20-25 chaetae; posteriorly unscaled, with about 40 chaetae; lateral flap unscaled, each side with about 25 chaetae. Tenaculum with $4+4$ teeth, unscaled, anterior face with 1 chaeta about as long as rami (Fig. 5D). Ratio manubrium: dens: mucro= 2.0-2.2:2.9-3.2:1.0. Manubrium ventrally with only scales, laterally with scales and 8 chaetae, proximal 2 chaetae small and slender, distal 6 chaetae distinctly stronger; dorsal scales absent; each dorsal chaetal stripe with about 70 chaetae in different sizes, without blunt chaetae; pseudopores 5-6 on each side (Fig. 5E); external corner chaeta as large as small chaetae in chaetal stripe (Fig. 5F). Dental spines formula $4 / 3$, I, a small pointed scale present ventrally to basal spines; all spines with several large denticles near base, superficial texture different between dorsal and ventral sides: dorsally with only fine longitudinal ribs, ventrally with longitudinal ribs and distal tiny serrations (Fig. 5G). The largest spine about 0.1 times as long as dens. Dens dorsally with feather-like chaetae between ordinary chaetae. Stripe of feather-like chaetae starting from centre of middle subsegment of dens, ending a short distance to the apex of dens. Ventral side of dens covered by scales, several chaetae present apically. Mucro elongated and multisetaceous, with two dorsal lamellae; both basal teeth with proximal lamellae, outer tooth without toothlet; apical tooth elongated, stronger than subapical tooth; intermediate teeth absent (Fig. 5H).

\section{Remarks}

Among Tomocerina, the new species is similar to those of the minuta group in its small body size, grey body colour and shape of mucro, but is different from them mainly in the chaetotaxy and the shape of dental spines. It is similar to Tomocerina purpurithora Liu, Hou \& Li, 1999 from Sichuan Province, China in the shape of dental spines, but is different from the latter mainly in the cephalic chaetotaxy, the number of tibiotarsal strong inner chaetae, the number of chaetae on tenaculum, the dental spines formula and the number of teeth on mucro (Table 1). Tomocerina annamitica sp. nov. is the southernmost distributed species of Tomocerina, a genus mostly diversified in northern temperate to cold temperate regions. However, so far Tomocerina is poorly defined by only a minute character: the absence of corner toothlet on outer basal tooth of mucro. In this respect, the presence of only $1+1$ bothriotricha on Th. II of Tomocerina annamitica sp. nov. might provide an interesting character, while most other species of Tomocerinae have $2+2$. Investigation on this character in different groups of Tomocerinae is in progress to assess its phylogenetic value. 


\section{Discussion}

Tomoceridae are mostly distributed in the northern temperate areas, with a diversity peak in eastern Palearctic: Japan (Yosii 1967), Korea (Lee 1975), Russian Far-East (Martynova 1977) and China (Ma 2004). The diversity rapidly decreases towards southern regions, but this family is still present in the caves or at high altitude of the subtropics (southern China and Vietnam, Denis 1929; Yu et al. 2014; Yu \& Deharveng 2015; Nepal, Yosii 1966), or even at high altitude in tropical regions (Sumatra, Oudemans 1891; Vietnam, Denis 1948). Before this study, all three species of Tomoceridae known from Vietnam belong to Tomocerus. The fourth species added here, Tomocerina annamitica sp. nov., belongs to another genus which had so far a single species distributed south of $30^{\circ} \mathrm{N}$ (Tomocerina simplex Yosii, 1966 from Nepal). Unexpectedly, the new species was frequent in litter and soil as low as $1000 \mathrm{~m}$ of elevation. Besides Tomocerus ocreatus and Tomocerina annamitica sp. nov., at least one other species is probably present in our collections from southern Annamitic Cordillera, confirming its status of southern extension for Palaearctic fauna of springtails (Deharveng \& Bedos 2000).

\section{Acknowledgements}

We thank Dr. Anne Bedos from MNHN Paris who helped to collect specimens in Lang Bian, Bi Doup and Hon Ba, Truong Quang Tam and Nguyen Tran Vy from Institute of Tropical Biology of Ho Chi Minh City who organized the field trip and the staff of the Bi Doup-Nui Ba National Park. Field work in Hon Ba was carried out in the frame of a project with the Nature Reserve, and supported by a French grant of the Agence Nationale de la Recherche under the LabEx ANR-10-LABX-0003-BCDiv. Laboratory work in NJAU was supported by the National Natural Science Foundation of China (41501056), the Fundamental Research Funds for the Central Universities (KJQN201668) and the Scientific Grant for Post-doctors of Jiangsu Province (1402054C).

\section{References}

Bellinger P.F., Christiansen K.A. \& Janssens F. 1996-2015. Checklist of the Collembola of the World [online]. Available from http://www.collembola.org [accessed 20 Mar. 2015]

Chiba S. 1968. Collembola of the Mt. Hakkoda area. I. Family Tomoceridae. The Science Reports of the Hirosaki University 15: 24-26.

Christiansen K. 1964. A revision of the Nearctic members of the genus Tomocerus (Collembola: Entomobryidae). Revue d'écologie et de biologie du sol 1: 668-675.

Deharveng L. \& Bedos A. 2000. Vietnura caerulea new genus, new species, from Vietnam: first record of the palaearctic tribe neanurini in tropical Asia (Collembola: Neanuridae). The Raffles Bulletin of Zoology 48 (2): 209-214.

Denis J.R. 1929. Notes sur les collemboles récoltés dans ses voyages par le Prof. F. Silvestri. Bollettino del Laboratorio di Zoologia generale e Agraria della R. Scuola Superiore d'Agricoltura in Portici 22: 166-180.

Denis J.R. 1948. Collemboles d'Indochine. Notes d'Entomologie Chinoise 12: 183-311.

Fjellberg A. 2007. The Collembola of Fennoscandia and Denmark. Part II. Entomobryomorpha and Symphypleona. Fauna Entomologica Scandinavica 42, Brill, Leiden. http://dx.doi.org/10.1163/ ej.9789004157705.i-265

Folsom J.W. 1913. North American spring-tails of the sub-family Tomocerinae. Proceedings of the United States National Museum 46: 451-472. http://dx.doi.org/10.5479/si.00963801.46-2037.451

Goto H.E. 1956. Architomocerura Denis, 1931 (Collemb., Isotomidae) an immature stage of Tomocerus Nicolet, 1841 (Collemb., Tomoceridae). Entomologist's Monthly Magazine 42: 49-51. 
YU D. et al., Tomoceridae from southern Annamitic cordillera

Krantz G.W. 1978. A manual of Acarology. Second edition. Oregon State University Bookstore, Inc. Corvallis.

Lee B.H. 1974. Étude de la faune coréenne des insectes Collemboles. V. Inventaire des grottes de corée et étude sur les Tomoceridae cavernicoles avec la description d'une nouvelle espèce. Annales de Spéléologie 29: 403-418.

Lee B.H. 1975. Étude de la faune coréenne des Insectes Collemboles. VI. Sur la famille des Tomoceridae, édaphiques, avec la description de quatre nouvelles espèces et d'une nouvelle sous-espèce. Bulletin du Muséum national d'Histoire naturelle, série 3 317: 945-961.

Liu Y.Q., Hou D.B. \& Li Z.C. 1998. A checklist of Collembola species from China. Journal of Southwest Agricultural University 20: 125-131.

Lubbock J. 1873. Monograph of the Collembola and Thysanura. Ray Society, London. http://dx.doi. org/10.5962/bhl.title. 11583

Ma Y.T. 2004. Taxonomic study on the genera Dicranocentrus and Entomobrya and the family Tomoceridae from China (Insecta: Apterygota). PhD thesis, Nanjing University, China.

Martynova E.F. 1977. Springtails of the family Tomoceridae (Collembola) from the fauna of the Far East. Insect Fauna of the Far East 46: 3-16.

Oudemans J.T. 1891. Apterygota des Indischen Archipels. Zoologische Ergebnisse einer Reisen in Niederländische Ost-Indien 1: 73-91.

Stach J. 1964. Materials to the knowledge of Chinese Collembolan Fauna. Acta Zoologica Cracoviensia 9: 1-26.

Stach J. 1965. On some Collembola of North Vietnam. Acta Zoologica Cracoviensia 10: 346-372.

Uchida H. 1953. The insect fauna of Mt. Ishizuchi and Omogo Valley, Iyo, Japan. The Collembola. Transactions of the Shikoku Entomological Society 4: 1-6.

Uchida H. \& Chiba S. 1958. Studies on the development of Tomocerus minutus Tullberg (Insecta: Collembola). I. On the postembryonic development. Zoological Magazine 67: 242-248.

Yosii R. 1956. Monographie zur Höhlencollembolen Japans. Contributions from the Biological Laboratory Kyoto University 3: 1-109.

Yosii R. \& Lee C.E. 1963. On some Collembola of Korea, with notes on the genus Ptenothrix. Contributions from the Biological Laboratory Kyoto University 15: 1-37.

Yosii R. 1966. Collembola of Himalaya. Journal of the College of Arts and Sciences, Chiba University 4: 461-531.

Yosii R. 1967. Studies on the Collembolan family Tomoceridae, with special reference to Japanese forms. Contributions from the Biological Laboratory Kyoto University 20: 1-54.

Yosii R. 1969. Collembola-Arthropleona of the IBP-station in the Shiga Heights, central Japan, I. Bulletin of the National Science Museum Tokyo 12: 531-556.

Yosii R. 1977. Critical check list of the Japanese species of Collembola. Contributions from the Biological Laboratory Kyoto University 25: 142-170.

Yu D.Y., Zhang F. \& Deharveng L. 2014. A peculiar cave species of Tomocerus (Collembola, Tomoceridae, Tomocerinae) from Vietnam, with a discussion of the postantennal organ and prelabral chaetae in Tomocerinae. ZooKeys 408: 61-70. http://dx.doi.org/10.3897/zookeys.408.7030 
Yu D.Y. \& Deharveng L. 2015. The first eyeless species of Tomocerus from China (Collembola, Tomoceridae) with notes on genera Tomocerus and Pogonognathellus. Zootaxa 3914 (2): 175-184. http://dx.doi.org/10.11646/zootaxa.3914.2.7

Zhang F., Yu D.Y., Luo Y.Z., Ho S.Y.W., Wang B.X. \& Zhu C.D. 2014. Cryptic diversity, diversification and vicariance in two species complexes of Tomocerus (Collembola, Tomoceridae) from China. Zoologica Scripta 43 (4): 393-404. http://dx.doi.org/10.1111/zsc.12056

Manuscript received: 17 October 2015

Manuscript accepted: 6 November 2015

Published on: 19 February 2016

Topic editor: Koen Martens

Desk editor: Charlotte Thionois

Printed versions of all papers are also deposited in the libraries of the institutes that are members of the EJT consortium: Muséum national d'Histoire naturelle, Paris, France; Botanic Garden Meise, Belgium; Royal Museum for Central Africa, Tervuren, Belgium; Natural History Museum, London, United Kingdom; Royal Belgian Institute of Natural Sciences, Brussels, Belgium; Natural History Museum of Denmark, Copenhagen, Denmark. 